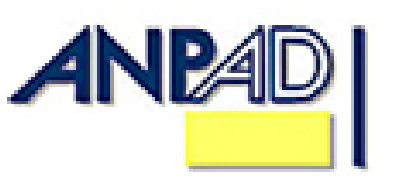
Disponível em
http://www.anpad.org.br/rac
RAC, Rio de Janeiro, v. 19, $2^{\text {a }}$ Edição Especial, art. 4, pp. 178-196, Agosto 2015 http://dx.doi.org/10.1590/1982-7849rac20151579
$(\mathrm{cc}) \mathrm{EY}$

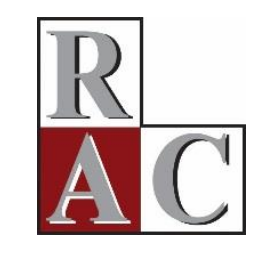

\title{
Quando Forças Opostas Aumentam a Intenção de Compra? Foco Motivacional e Mensagens de Comunicação
}

When Do Opposing Forces Increase Willingness to Buy? Regulatory Motivational Focus and Ad Messages

\author{
Danielle Mantovani \\ Universidade Federal do Paraná - UFPR/PPGADM \\ Mariana Monfort Barboza \\ Universidade Federal do Paraná - UFPR/PPGADM \\ Juan José Camou Viacava \\ Universidade Federal do Paraná - UFPR/PPGADM \\ Paulo Henrique Muller Prado \\ Universidade Federal do Paraná - UFPR/PPGADM
}




\title{
Resumo
}

A teoria do ajuste regulatório estabelece que os consumidores se baseiam no seu foco motivacional (prevenção vs. promoção) para fazer avaliações de consumo e tomar decisões. Normalmente, os consumidores preferem produtos apresentados com o mesmo foco motivacional que o seu. Estudos anteriores mostram que esse padrão de preferência ocorre quando não somos motivados a processar informações. Porém, este estudo propõe que em situações que exigem maior esforço cognitivo, nas quais o consumidor precisa de mais informações para decidir, dada a dificuldade da tarefa, as mensagens que apresentem foco regulatório motivacional contrário ao do consumidor serão mais persuasivas. Dessa forma, os consumidores mostrarão maior intenção de comprar produtos apresentados em foco de não ajuste regulatório quando forem previamente expostos a tarefas difíceis, que exigem maior esforço cognitivo. O experimento $1(n=257)$ mostra evidências da moderação da dificuldade da tarefa sobre a persuasão de mensagens de anúncios, enquanto o experimento $2(n=144)$ apresenta este efeito em intenções de compra. Assim, nem sempre as informações compatíveis com o foco motivacional do consumidor são as mais bem avaliadas. Quando maior esforço cognitivo é requerido, mensagens de não ajuste com o foco regulatório motivacional poderão ser mais persuasivas.

Palavras-chave: foco regulatório; ajuste (vs. não ajuste) regulatório; dificuldade da tarefa; processamento de informações.

\begin{abstract}
The regulatory focus theory suggests that consumers rely on their motivational focus (prevention vs. promotion) for their evaluations and decisions. Usually consumers prefer products that match their personal motivational focus. Previous studies show that this pattern preferably occurs when we are not motivated to process information. However, this study suggests that situations that require greater cognitive effort, in which the consumer needs more information to decide because of the difficulty of the task, messages that have an opposing motivational regulatory focus will be more persuasive. When consumers have not been previously exposed to difficult tasks which require more cognitive effort, their willingness to buy products presented in ways that match their regulatory fit will increase. Experiment $1(n=257)$ showed evidence that task difficulty has a moderating role in ad-message persuasiveness. Experiment $2(n=144)$ presents the same moderating effect of task difficulty on the regulatory fit (vs unfit) phenomenon for willingness to buy. Therefore, not all information compatible with consumer motivational focus is well evaluated. When greater cognitive effort is required, unfit messages may be more persuasive.
\end{abstract}

Key words: regulatory focus; regulatory (un)fit; task difficulty; information processing. 


\section{Introdução}

Considere dois anúncios para divulgar a marca de filtro solar SunSkin. O primeiro apresenta a seguinte mensagem: Aproveite o verão com SunSkin. Já o segundo argumenta: Fique segura neste verão com SunSkin. Com um anúncio que enfatiza atingir resultados positivos e outro que enfatiza a proteção, qual deles você acha que seria mais persuasivo? Estudos mostram que essa resposta depende do foco regulatório do consumidor.

A teoria do foco regulatório proposta por Higgins $(1997,2012)$ é uma das vertentes teóricas que explica o comportamento motivacional dos consumidores. Para esta teoria, o foco regulatório dos indivíduos pode ser considerado o mecanismo hedônico pelo qual os indivíduos tendem a buscar as ações que lhes remetem prazer e lhes evitam a dor ao serem executadas.

Essa teoria tem dois mecanismos atuantes: o de promoção (promotion), orientação para atingir resultados positivos, como por exemplo, fazer uma dieta objetivando melhorar o bem-estar; e o de prevenção (prevention), orientação para evitar resultados negativos, como por exemplo, fazer uma dieta para prevenir doenças advindas do excesso de peso, como pressão alta e diabetes) (Higgins, 1997, 2012; Higgins et al., 2001).

Dessa forma, de acordo com a teoria do Foco Regulatório, os consumidores com foco em promoção apresentariam maior intenção de compra ao serem apresentados a anúncios com mensagens de promoção, como por exemplo, Aproveite o verão com SunSkin. Por outro lado, os consumidores focados em prevenção seriam mais persuadidos por argumentos com foco de prevenção, entre eles, Fique segura neste verão com SunSkin (Aaker \& Lee, 2001; Cesario \& Higgins, 2008; Higgins \& Scholer, 2009). Esse fenômeno é chamado de ajuste regulatório. O ajuste regulatório é a congruência entre a orientação motivacional do indivíduo, seja de promoção ou prevenção, e a estratégia para atingir suas metas. A relação entre essa orientação e a maneira como uma meta é perseguida normalmente afeta as avaliações de consumo e, portanto, o comportamento (Higgins, 2012). Por isso, caso o consumidor esteja com foco mais voltado para atingir resultados positivos, provavelmente a mensagem que enfatiza aproveitar o verão será a preferida. Entretanto, se a meta do indivíduo é evitar problemas de exposição ao sol, a mensagem sobre segurança será a mais persuasiva.

Esse ajuste ocorre porque as informações são compatíveis com a orientação motivacional do consumidor naquele momento. Por isso, é mais fácil processar e compreender estas informações por serem consistentes com a orientação regulatória do indivíduo.

Embora o fenômeno do ajuste regulatório predomine na literatura, novos estudos propõem que o não ajuste regulatório pode ser mais persuasivo em relação ao ajuste de acordo as com condições situacionais (Higgins \& Scholer, 2009; Lee, 2009; Tam \& Spanjol, 2012; Vaughn et al., 2006). Nesse caso, indivíduos com foco em promoção apresentariam maior intenção de compra quando apresentados a anúncios com mensagens de prevenção, sendo o oposto verdadeiro para indivíduos com foco em prevenção.

Alguns estudos (Harding, Lisjak, \& Lee, 2009; Lee, 2009) demonstraram que em condições nas quais os indivíduos estão suficientemente motivados a processar novas informações, a condição de não ajuste regulatório é mais favorável. Uma das formas de estimular o processamento de informações é fazê-lo em condições de dificuldade da tarefa. Em situações difíceis, os indivíduos tendem a empregar o máximo de esforços e gastar mais recursos processando informações, numa tentativa de reforçar sua confiança com relação ao julgamento e avaliação das informações a serem processadas (Harding et al., 2009; Higgins \& Scholer, 2009; Lee, 2009). Esse comportamento ocorre porque as pessoas gastam mais tempo e recursos processando informações que não são consistentes com suas atitudes (Norris, 2007).

Assim, em situações em que o consumidor é motivado a processar mais informações, mensagens que apresentem foco motivacional contrário ao seu serão mais persuasivas, e as intenções de compra 
serão maiores em relação àquelas que apresentam ajuste regulatório com o foco motivacional do consumidor.

O presente estudo mostra ainda que o efeito moderador da dificuldade da tarefa se sobrepõe ao alto envolvimento do consumidor. O estudo de Harding, Lisjak e Lee (2009) mostrou que, sob condições de alto envolvimento, os participantes apresentaram maior propensão de compra para anúncios de não ajuste em relação àqueles em situações de ajuste. O contrário ocorreu para situações de baixo envolvimento.

Entretanto, este estudo mostra que mesmo sob condições de alto envolvimento, a dificuldade (vs. facilidade) da tarefa irá moderar as avaliações de acordo com o tipo de ajuste. Assim, o envolvimento por si só pode não ser suficiente para induzir diferentes níveis de processamento cognitivo e, por consequência, alterar as avaliações dos consumidores.

Nesse contexto, este estudo contribui com a área de comportamento do consumidor, especificamente com a psicologia do consumo, ao explicar como a dificuldade da tarefa pode influenciar na persuasão dos consumidores com base em seu foco motivacional, seja ele evitar perdas ou conquistar ganhos.

\section{Teoria do Foco Regulatório}

Há um processo básico na busca de metas conhecido por Teoria do Foco Regulatório (Regulatory Focus Theory) (Higgins, 1997). Essa teoria propõe que as pessoas têm diferentes orientações regulatórias durante a busca de suas metas. Assim, há alguns mecanismos atuantes no processo motivacional de perseguição da meta, como os mecanismos de promoção e prevenção (Aaker \& Lee, 2001; Higgins, 2002). O foco promocional envolve uma sensibilidade a resultados positivos (sua presença e ausência), enquanto o foco de prevenção envolve uma sensibilidade a resultados negativos (sua presença e ausência) (Higgins, 2002).

A Teoria do Foco Regulatório é cada vez mais utilizada para explicar uma gama de fenômenos de consumo (processos psicológicos e comportamentais). O foco em promoção enfatiza um eu ideal, na condição de esperanças e aspirações dos indivíduos, como por exemplo, fazer dieta para se sentir mais bonita e confiante. Já o foco em prevenção enfatiza o eu obrigatório, na condição de reflexo dos deveres e obrigações dos indivíduos, como por exemplo, fazer dieta para evitar doenças (Haws, Dholakia, \& Bearden, 2010).

Uma premissa básica da Teoria do Foco Regulatório é que os indivíduos estão mais preocupados com informações relevantes para o foco regulatório no qual se encontram, sendo, portanto, os atributos compatíveis com tal foco, provavelmente mais bem avaliados e considerados mais relevantes na escolha (Chernev, 2004). Assim, indivíduos com foco em prevenção regulam suas atitudes e seu comportamento no intuito de conseguir proteção e segurança, enquanto aqueles com foco em promoção regulam suas atitudes e comportamentos para aspirações e realizações (Higgins, 1997, 2012).

O impacto do foco regulatório sobre o comportamento dos indivíduos em situações de consumo tem sido bastante estudado nos últimos anos e apresentado contribuições relevantes para avaliar como os consumidores definem suas metas e motivações de consumo. Para Aaker e Lee (2001), dependendo do foco regulatório dominante, os consumidores respondem diferentemente às comunicações de marketing.

Diversos estudos analisaram o impacto do foco regulatório sobre o comportamento dos consumidores. Esses estudos mostram como a manipulação do foco regulatório impacta diretamente nas preferências e avaliações dos produtos pelos consumidores (Dholakia, Gopinath, Bagozzi, \& Nataraajan, 2006; Mogilner, Aaker, \& Pennington, 2008; Werth \& Foerster, 2007), na persuasão de mensagens de anúncios (Lee \& Aaker, 2004), na avaliação de anúncios (Florack, Ineichen, \& Bieri, 2009; Pham \& 
Avnet, 2009), no nível de interpretação e busca das informações e atitudes (Lee, Keller \& Sternthal, 2010; Pham \& Chang, 2010), entre outros aspectos.

Todos esses estudos consideraram a perspectiva do ajuste regulatório na relação entre o foco motivacional do indivíduo e as avaliações posteriores de consumo.

\section{Ajuste e não ajuste regulatório}

O ajuste regulatório para o indivíduo é a derivação de utilidade adicional até o ponto em que os meios usados para perseguir uma determinada meta são compatíveis com o foco regulatório desse indivíduo (Vaughn et al., 2006). Nesse sentido, os indivíduos experimentam um ajuste regulatório quando adotam estratégias para perseguir uma meta ou quando desenvolvem atividades que estejam de acordo com sua orientação regulatória (Aaker \& Lee, 2006; Avnet, Laufer, \& Higgins, 2013).

Para Higgins (2002), em condições de ajuste regulatório, as avaliações das pessoas sobre decisões passadas ou sobre a perseguição de metas futuras serão mais positivas, assim como mais positivo será o valor atribuído para produtos escolhidos sob essas condições, pois o ajuste aumenta a percepção do indivíduo de que ele utilizou uma estratégia correta.

Uma premissa básica da Teoria do Foco Regulatório é que os indivíduos estão mais preocupados com informações relevantes para o foco regulatório em que se encontram. Portanto, os atributos compatíveis com tal perspectiva provavelmente serão mais bem avaliados e considerados mais relevantes na escolha (Chernev, 2004).

Vários estudos têm demonstrado que, em situações de ajuste regulatório, os indivíduos serão mais persuadidos pelas mensagens (Higgins \& Scholer, 2009). Por exemplo, os participantes expostos a mensagens que se encaixem em sua orientação regulatória aumentam o consumo de frutas e legumes (Cesario, Grant, \& Higgins, 2004), usam mais protetor solar (Lee \& Aaker, 2004) e aumentam a realização de atividades físicas (Latimer et al., 2007).

Tal efeito pode ser explicado pela experiência de feeling right (Appelt, Zou, \& Higgins, 2010; Avnet, Pham, \& Stephen, 2012; Pham \& Avnet, 2009; Pham, Lee, \& Stephen, 2012). Por meio desta experiência, os indivíduos percebem determinadas mensagens como as mais corretas. Indivíduos em estado de promoção sentem que as mensagens com o mesmo foco são as mais corretas, pois utilizam essa percepção de promoção como informação para avaliar a mensagem, o mesmo sendo observado para os indivíduos em foco de prevenção em relação às mensagens com argumentos de prevenção.

Porém, é interessante observar que o ajuste entre o foco regulatório e as informações só ocorre para situações de baixo envolvimento, enquanto o não ajuste tem apresentado melhores resultados sobre avaliações e comportamento de compra em condições de alto envolvimento (Harding et al., 2009; J. Wang \& Lee, 2006). Desse modo, estudos recentes sugerem uma nova perspectiva para discutir os limites do ajuste regulatório (Higgins \& Scholer, 2009; Lee, 2009; Tam \& Spanjol, 2012; Vaughn et al., 2006).

A explicação para esse efeito pode ser encontrada no estudo de Forster, Higgins e Werth (2004). Para os autores, sob condições de alto envolvimento, as pessoas dão mais atenção e têm maior memória para informações inconsistentes. Em outro estudo, J. Wang e Lee (2006) manipularam o envolvimento, apresentando ambas as informações de produto (de ajuste e não-ajuste) aos indivíduos e observaram que em condições de baixo envolvimento, estes indivíduos procuravam informações e produtos com características que se ajustassem a seu foco regulatório. Entretanto, quando os indivíduos eram motivados a processar informação, o efeito do ajuste na busca por informações era dissipado.

Para Vaughn et al. (2006), o não ajuste regulatório tende a motivar a melhoria no desempenho quando os indivíduos estão preocupados com a tarefa, pois, nesta situação, eles tendem a empregar maiores esforços pelo sentimento de preocupação com sua realização, até sentirem que estão fazendo 
um bom trabalho. Ao contrário, quando os indivíduos apresentam satisfação e prazer na execução de uma tarefa, o ajuste tende a ter maior efeito para o aumento da motivação na sua execução.

Harding et al. (2009) verificaram, ao manipular tipos de ajuste (ajuste vs. não ajuste) sob alto envolvimento, que respondentes em não ajuste teriam se esforçado mais, engajando-se mais no processamento cognitivo de avaliação de marcas, tendo observado seu resultado em avaliações mais positivas sobre estas marcas.

Assim, neste estudo, propõe-se que o efeito advindo do alto envolvimento pode ser alterado quando os indivíduos recebem um priming de dificuldade da tarefa, sendo, portanto, mais fortemente influenciados a processar informações em comparação com aqueles que não recebem tal estímulo, mas ainda assim estarão em situação de alto envolvimento.

Estudos que discutem o engajamento do consumidor propuseram que a dificuldade em realizar uma tarefa e suas condições de engajamento interferem na perseguição de metas (Higgins \& Scholer, 2009; Lee, 2009). Tarefas difíceis demandam do indivíduo maior processamento de informações em virtude do alto esforço cognitivo (Faro, Heller, \& Irmak, 2011; Reinhard \& Dickhauser, 2011; Rice et al., 2012). Essas tarefas podem ou não ser relacionadas a situações de compra, mas disparam maior processamento de informações.

No estudo de Faro, Heller e Irmak (2011), por exemplo, a dificuldade da tarefa foi manipulada pela resolução de anagramas e mensurada pela variação do seu tamanho. Participantes na condição de baixa dificuldade solucionavam anagramas de quatro letras; os de dificuldade média, anagramas de cinco letras e os da condição de alta dificuldade, anagramas de seis letras.

Ainda, no estudo de Nordgren e Dijksterhuis (2009), a complexidade e a dificuldade da tarefa foram capazes de diminuir a consistência de deliberações, resultando em inconsistências de pesos nas informações e no processamento cognitivo, além de um tempo significativamente maior para formar avaliações. O efeito que a realização de uma compra mais difícil, como a compra de um apartamento, por exemplo, teria sobre o comportamento do indivíduo, será o mesmo, visto que o consumidor precisa buscar informações, comparar opções, definir critérios de escolha, e todo esse esforço leva a um maior processamento de informações (Bettman, Luce, \& Payne, 1998).

Dessa forma, quando o envolvimento for alto, mas a percepção de esforço cognitivo requerido for baixo, o ajuste regulatório, pela sua sensação de feeling right, pode tornar-se um meio rápido, atrativo e mais congruente para avaliações de consumo. Entretanto, quando os indivíduos estão em alto envolvimento e enfrentam alta dificuldade percebida, tendem perceber informações que não se ajustam (não ajuste regulatório) como mais relevantes.

Assim, caso um consumidor em alto envolvimento esteja com foco voltado para evitar resultados negativos (foco em prevenção) e tiver que realizar uma tarefa mais fácil, ele será motivado a processar favoravelmente informações que se ajustem a seu foco de prevenção, enquanto um consumidor em promoção terá o mesmo feito para anúncios de promoção. Contudo, ao estar motivado e enfrentar dificuldades para avaliar um anúncio, argumentos que se ajustam a seu foco regulatório serão menos persuasivos por parecerem mais fáceis em relação àqueles argumentos com foco regulatório contrário ao do indivíduo. Neste sentido, a dificuldade da tarefa irá moderar a relação entre o foco regulatório do consumidor e o foco regulatório preponderante em situações de consumo sob alto envolvimento. Em condições que exigem maior processamento de informações, como as de alto esforço cognitivo, anúncios de não ajuste regulatório serão mais persuasivos. Já em condições de baixo esforço cognitivo, os anúncios de ajuste regulatório serão mais persuasivos. Neste contexto, é proposta a seguinte hipótese:

Hipótese: A influência do ajuste e do não ajuste regulatório sobre as avaliações de mensagens de anúncios sob condições de alto envolvimento será moderada pela dificuldade da tarefa. Quando a dificuldade for baixa, as avaliações serão mais positivas para anúncios de ajuste, enquanto a alta dificuldade influenciará nas avaliações mais positivas para anúncios de não ajuste regulatório. 


\section{Método}

O método de pesquisa adotado é o de abordagem quantitativa, de classificação causal e de natureza experimental, conforme proposta de Shadish, Cook e Campbell (2002). Em todos os estudos, a coleta de dados foi do tipo não probabilística por conveniência, mas a alocação dos respondentes em cada uma das condições foi feita de maneira aleatória. As amostras foram compostas por estudantes de graduação, de todos os semestres, dos cursos de Administração e Economia, da Universidade Federal do Paraná.

A participação na pesquisa foi voluntária, e os respondentes receberam créditos de atividades complementares para participar do estudo. Os respondentes do experimento 1 não participaram novamente no experimento 2 , dada a necessidade de desconhecimento do propósito da pesquisa.

Para garantir a validade de face, o roteiro de cada um dos estudos foi encaminhado a três pesquisadores experientes no uso da metodologia experimental. A validade de conteúdo e a de construto para as variáveis independentes, foco e ajuste regulatório foram garantidas pela utilização de manipulações já validadas em estudos anteriores (Dholakia et al., 2006; Fishbach \& Dhar, 2005; Higgins, 1997). A mesma situação ocorreu para a variável moderadora, dificuldade da tarefa, cuja manipulação foi originalmente proposta no estudo de Faro et al. (2011).

O experimento 1 teve o objetivo de investigar o efeito moderador da dificuldade da tarefa na relação entre o ajuste (vs. não ajuste) regulatório e a avaliação dos consumidores de mensagens de anúncios em situações subsequentes de consumo. $\mathrm{O}$ experimento 2 teve dois objetivos: o primeiro foi o de mostrar que os efeitos encontrados no experimento precedente eram consistentes e o segundo, de minimizar algumas das limitações verificadas no experimento 1 .

\section{Experimento 1}

\section{Amostra, design e procedimento}

A amostra foi composta por 275 estudantes de graduação (47,6\% mulheres, com idade média de 22,6 anos) com between subjects design 2 (priming foco promoção vs. prevenção) x 2 (baixa vs. alta dificuldade) x 2 (anúncio foco promoção vs. prevenção), tendo sido os respondentes alocados aleatoriamente em uma das oito condições.

Uma variável Tipo de Ajuste (ajuste vs. não ajuste) foi criada combinando o priming de foco regulatório com o foco da mensagem, como em Higgins (1997, 2004), Dholakia, Gopinath, Bagozzi e Nataraajan (2006) e Harding et al. (2009). O prime cognitivo é uma manipulação para induzir um comportamento específico, como por exemplo, o foco regulatório que, neste estudo, é utilizado como sinônimo de uma manipulação para induzir este mesmo foco regulatório. Para detalhes sobre o efeito de priming, ver Laran, Janiszewski e Cunha (2008).

Desta forma, os participantes que viram a mensagem condizente com seu foco regulatório foram classificados em Ajuste e aqueles que avaliaram a mensagem oposta ao seu foco regulatório foram classificados em condição de Não Ajuste regulatório, tendo sido analisadas as variáveis com um design 2 (ajuste vs. não ajuste) x 2 (baixa vs. alta dificuldade).

Os respondentes, em forma de cover story, foram convidados a participar de uma pesquisa envolvendo três partes não relacionadas: a primeira, sobre Objetivos Acadêmicos e Profissionais; a segunda, sobre capacidade de resolução de problemas e a terceira, sobre avaliações de mensagens de anúncios. Todas as pesquisas foram realizadas em sala de aula, com papel e caneta. A duração média de todas as etapas foi de aproximadamente 30 minutos. 
$\mathrm{Na}$ primeira parte, os participantes foram aleatoriamente alocados nas condições de foco regulatório. Na condição de prevenção, foram solicitados a escrever seus principais deveres e obrigações envolvendo seu futuro acadêmico e profissional, e depois listar pelo menos cinco estratégias que eles poderiam utilizar para evitar que algo lhes impedisse de realizá-las (Dholakia et al., 2006).

Em seguida, foram solicitados a elaborar uma redação sobre Prevenção é a melhor forma de evitar males (Higgins, 1997, 2004). Os participantes na condição de promoção foram instruídos a escrever suas principais esperanças e aspirações envolvendo seu futuro acadêmico e profissional e, em seguida, listar pelo menos cinco estratégias para ajudar na realização dos objetivos (Dholakia et al., 2006). Já o tema da redação foi Sucesso na vida é determinado pela ação, e não pela inércia (Higgins, 1997, 2004).

Após finalizarem essa primeira etapa, os respondentes foram convidados a participar de uma segunda pesquisa. Primeiramente, todos os participantes receberam um priming para gerar alto envolvimento. Essa manipulação foi feita com base no estudo de Harding et al. (2009), que consiste em criar um envolvimento por uma frase, no caso, em tela, "Vocês fazem parte de um grupo muito pequeno, uma amostra selecionada minuciosamente e suas respostas à pesquisa serão analisadas individualmente" (p. 455). Essa manipulação serviu para garantir que todos os participantes se sentissem envolvidos na tarefa, evitando possíveis diferenças no comportamento dos respondentes.

Posteriormente, os participantes receberam a manipulação de dificuldade da tarefa (baixa dificuldade vs. alta dificuldade). Foi utilizada a proposta de Faro et al. (2011), que consiste em tarefas de resolução de anagramas de tamanhos diferentes com tempo máximo de dez minutos. Participantes na condição de baixa dificuldade deveriam solucionar dez anagramas de quatro letras, como por exemplo: $\mathrm{ROMA}=\mathrm{AMOR}$; e participantes na condição de alta dificuldade deveriam solucionar anagramas de seis letras, como por exemplo: OCLSAE = ESCOLA. Ao final, os respondentes informaram o quão difícil foi realizar esta tarefa em uma escala de sete pontos (1= muito fácil; $7=$ muito difícil). Como variável de controle, foi mensurado ainda o envolvimento na realização da tarefa $(1=$ pouco envolvido; 7 = muito envolvido).

$\mathrm{Na}$ terceira parte do estudo, os respondentes foram convidados a participar de outra pesquisa não relacionada às anteriores, sobre avaliação de mensagens de anúncios publicitários. Para a manipulação de foco regulatório nas mensagens, os participantes receberam aleatoriamente um dos dois anúncios abaixo, tendo por base mensagens de prevenção ou de promoção, de uma marca $\mathrm{X}$ de suco de uva, adaptado de Aaker e Lee (2001). Os anúncios são descritos a seguir:

\section{(Anúncio com foco em Promoção)}

\section{Suco de Uva da Marca X}

Estudos médicos preliminares sugerem que beber suco de uva pode contribuir para dar mais disposição e energia! Diversas evidências sugerem que uma dieta rica em Vitamina C e Ferro leva a altos níveis de energia para realizar as atividades diárias. De acordo com uma pesquisa do Departamento Brasileiro de Agricultura, o suco de uva da Marca X contém três vezes mais Vitamina $\mathrm{C}$ e Ferro que os demais sucos. As uvas utilizadas na fabricação do suco da Marca X são colhidas somente quando a fruta atinge seu melhor sabor, por isso o Suco de Uva da Marca $\mathrm{X}$ é tão saboroso quanto energizante. Além disso, beber suco da Marca X é muito prazeroso! Temos orgulho de afirmar que tudo relacionado à Marca $X$ tem os mais elevados padrões de qualidade em sabor, diversão e energia. (Aaker \& Lee, 2001, p. 36).

\section{(Anúncio com foco em Prevenção) \\ Suco de Uva da Marca X}

Estudos médicos preliminares sugerem que beber suco de uva pode contribuir para a melhoria das funções cardiovasculares. Diversas evidências sugerem que uma dieta rica em antioxidantes pode reduzir o risco de alguns tipos de câncer e de doenças do coração. De acordo com uma pesquisa do Departamento Brasileiro de Agricultura, o suco de uva da Marca X contém três vezes mais antioxidantes que os demais sucos. Os antioxidantes do suco de uva são atribuídos aos flavonoides 
contidos no suco, que ajudam a manter as artérias limpas para que o sangue possa circular com mais facilidade. Portanto, é saudável beber o suco de uva da Marca X!

Temos orgulho de afirmar que tudo relacionado à Marca $X$ tem os mais elevados padrões de qualidade em saúde e bem-estar. (Aaker \& Lee, 2001, pp. 36-37).

Depois de ler o anúncio, os respondentes avaliavam quanto consideravam a mensagem persuasiva (Quanto você considerou essa mensagem persuasiva?) em uma escala de sete pontos $(1$ = nada persuasiva; 7 = muito persuasiva), informando também o envolvimento na avaliação dos anúncios ( $1=$ pouco envolvido; 7 = muito envolvido).

Por fim, havia uma questão para verificar se os respondentes haviam percebido a relação entre as partes ou entre as pesquisas (hypothesis guessing check). Nenhum dos respondentes associou os dois estudos ou conseguiu adivinhar o propósito da pesquisa. Havia também duas questões demográficas (idade e gênero).

\section{Resultados}

A checagem da manipulação de foco regulatório de prevenção e promoção foi feita conforme Dholakia et al. (2006), de modo qualitativo. As afirmações dos respondentes na condição de promoção deveriam estar orientadas para alcançar resultados positivos, enquanto as respostas advindas da condição de prevenção deveriam estar orientadas para evitar resultados negativos. Nenhum caso precisou ser retirado da amostra por este motivo.

Para checar a percepção de dificuldade da tarefa, foram utilizadas duas mensurações. A primeira referia-se à dificuldade na resolução dos anagramas. Observou-se diferença significativa entre os grupos $\left[\mathrm{t}(273)=-18,390, \mathrm{p}<0,001, \mathrm{M}_{\text {altadificuldade }}=4,95 ; \mathrm{D} . \mathrm{P}=1,51, \mathrm{M}_{\text {baixadificuldade }}=1,93 ; \mathrm{D} \cdot \mathrm{P}=1,20\right]$, conforme desejado. Entretanto, verificou-se que 18 participantes $\left(6,5 \%\right.$ da amostra: $n_{\text {baixadificuldade }}=6 ; n_{\text {altadificuldade }}=$ 12) responderam à checagem no completo oposto da escala, por isto foram excluídos da amostra final ( $\mathrm{n}=257 ; 51,8 \%$ homens $)$ e das análises.

A checagem de número de acertos na resolução dos anagramas pelo teste $\mathrm{t}$ para amostras independentes teve o resultado desejado [t $(255)=17,48, \mathrm{p}<0,001, \mathrm{M}_{\text {baixadificuldade }}=9,47$; D.P $=0,87$, $\left.\mathrm{M}_{\text {altadificuldade }}=5,68 ; \mathrm{D} \cdot \mathrm{P}=2,31\right]$, com menos acertos para o grupo de alta dificuldade.

Para evitar um possível confound na interpretação dos resultados, os grupos de baixa e alta dificuldade da tarefa foram comparados em relação ao grau de envolvimento na realização da tarefa de resolução dos anagramas, além do envolvimento com a avaliação do anúncio. Não foram encontradas diferenças significativas para envolvimento da tarefa de resolução dos anagramas [ $\mathrm{t}(255)=-1,22, \mathrm{p}=$ $\left.0,226, \mathrm{M}_{\text {baixadificuldade }}=5,91 ; \mathrm{D} \cdot \mathrm{P}=6,10, \mathrm{M}_{\text {altadificuldade }}=6,10 ; \mathrm{D} \cdot \mathrm{P}=1,14\right]$. Já a média de envolvimento com a avaliação do anúncio foi ligeiramente mais baixa, porém ainda considerada alta para as condições analisadas e, o que é mais importante, sem diferenças entre os grupos [t $(255)=-1,29, \mathrm{p}=0,195$; $\mathrm{M}_{\text {baixadificuldade }}=4,78 ;$ D.P $\left.=1,44 ; M_{\text {altadificuldade }}=5,00 ; D \cdot P=1,22\right]$. Desta forma, são consideradas satisfatórias para analisar os resultados.

Para testar a hipótese de que a dificuldade da tarefa modera a relação entre o ajuste (vs. não ajuste) regulatório e a persuasão das mensagens de anúncios, uma ANOVA com o design 2 (dificuldade da tarefa: baixa vs. alta) por 2 (Ajuste vs. Não-ajuste) between subjects foi feita utilizando a persuasão como variável dependente. Houve efeito principal significativo da dificuldade da tarefa $[F(1,253)=$ $\left.8,44, p=0,004, \eta_{\mathrm{p}}{ }^{2}=0,032\right]$, mas não significativo para o tipo de ajuste $[F(1,253)=0,020, p=0,890]$. O efeito esperado de interação entre os níveis de dificuldade da tarefa e o tipo de ajuste sobre a persuasão da mensagem foi marginalmente significativo $\left[F(3,253)=2,770, p=0,097, \eta_{\mathrm{p}}{ }^{2}=0,011\right]$, conforme a Figura 1 apresenta. 


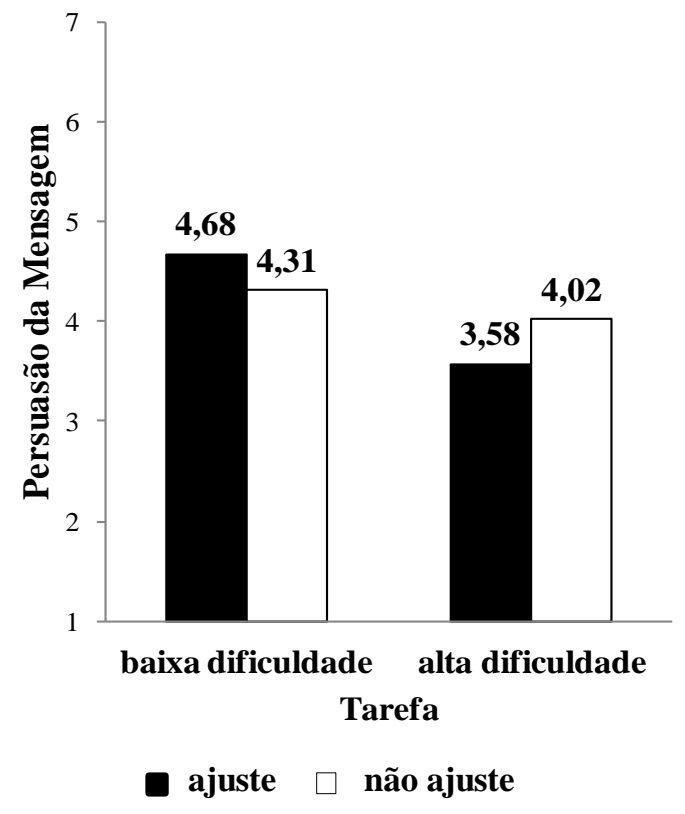

Figura 1. Persuasão da Mensagem em Função da Dificuldade da Tarefa

Comparações post hoc mostraram que a média de persuasão da mensagem na condição de baixa dificuldade, lado esquerdo da Figura 1, é maior para o grupo de ajuste $(M=4,68 ; D . P=1,73)$ do que de não-ajuste, mas não de forma significativa $(\mathrm{M}=4,31$; D.P $=1,91),(F(1,128)=1,289, p=0,256)$.

Além disso, não houve diferença significativa para a média de avaliação do anúncio na condição de alta dificuldade, lado direito da Figura 1, para ajuste $(M=3,58$; D.P $=1,86)$ e não ajuste $(M=4,02$; D.P $=2,14)(F(1,127)=1,479, p=0,226)$, ainda que para ambos os casos os resultados se deram no sentido da hipótese.

Comparações entre os grupos de ajuste, barras preenchidas, indicaram diferenças significativas $\left.\left[F(1,129)=11,989, p<0,001, \eta_{\mathrm{p}}{ }^{2}=0,086\right)\right]$. Esse resultado mostra que com o aumento da dificuldade, a persuasão do anúncio ajustado entre o foco motivacional do consumidor torna-se estatisticamente menor. Dentro do grupo de não ajuste, a diferença entre as condições de alta e baixa dificuldade não atingiu significância estatística $[F(1,126)=0,678, p=0,412]$.

\section{Discussão}

Os resultados do experimento 1 fornecem suporte inicial para a hipótese. A dificuldade da tarefa influenciou, ainda que marginalmente, na relação entre o ajuste regulatório do indivíduo e as informações de comunicação, afetando a avaliação da mensagem de comunicação. A alta dificuldade moderou os efeitos do ajuste sobre a persuasão do anúncio, conforme ilustrado na Figura 1.

O experimento 1 mostra indícios de que o impacto positivo do ajuste regulatório sobre as avaliações dos consumidores tende a ocorrer somente em situações de baixo esforço cognitivo. Estudos anteriores (Avnet et al., 2012; Avnet et al., 2013; Pham et al., 2012) também apresentaram evidências nesse sentido. Tais estudos explicam esse comportamento pelo efeito de feeling right (Pham \& Avnet, 2009). Devido à facilidade em processar informações, os consumidores seguem regras de decisão mais simples. Assim, informações compatíveis com seu foco motivacional despertarão o sentido de que é a mais correta.

Indivíduos em estado de promoção, focados em realizações e aspirações, avaliaram o anúncio com argumentos sobre energia e disposição de maneira mais positiva. Já os indivíduos em estado de prevenção, motivados a buscar segurança e evitar problemas, serão mais persuadidos por anúncios com foco em evitar doenças. 
Porém, esse padrão só é verdadeiro se os indivíduos não forem estimulados a processar informações. Assim, o presente estudo mostrou que na condição de dificuldade da tarefa, na qual o consumidor é estimulado a processar informações, o efeito do ajuste sobre a persuasão da mensagem do anúncio diminuiu, foi de $\mathrm{M}_{\text {ajuste_baixa dificuldade }}=4,68$ para $\mathrm{M}_{\text {ajuste_alta dificuldade }}=3,58$. Estudos anteriores evidenciam que em situações nas quais os consumidores são estimulados a processar informações, o impacto positivo do ajuste regulatório sobre a resposta de consumo tende a diminuir (ver Harding et al., 2009 - por exemplo). Assim, este estudo aponta mais uma condição na qual essa situação acontece, ou seja, em tarefas consideradas mais difíceis.

Uma contribuição adicional deste estudo é mostrar que a moderação da dificuldade da tarefa acontece também quando há alto envolvimento com a atividade. No estudo de Harding et al. (2009), os autores mostraram que os consumidores somente são estimulados a processar mais informações quando estão em alto envolvimento. No presente estudo, há evidências de que mesmo em alto envolvimento, se o consumidor não for estimulado a processar informações (condição de baixa dificuldade), o ajuste terá maior poder de persuasão em relação ao não ajuste.

Algumas limitações deste estudo devem ser relatadas. A principal delas foi que, apesar de haver efeito de interação entre tipo de ajuste e dificuldade da tarefa, nem todas as comparações post hoc foram significativas. Outra limitação foi a necessidade de excluir 18 participantes, o que representa uma porcentagem pequena, de 6,5\%, em relação à amostra uma porcentagem pequena de $6,5 \%$ em relação à amostra.

Nesse sentido, o segundo experimento apresenta um cenário diferente de avaliação de consumo, no intuito de minimizar as limitações do primeiro estudo e tornar mais robusto e consistente o efeito de moderação da dificuldade da tarefa.

\section{Experimento 2}

O experimento 2 buscou mostrar a consistência de efeitos encontrados no experimento 1. Dessa forma, pretende-se confirmar a hipótese de que o nível de dificuldade da tarefa seja capaz de moderar o impacto do ajuste (vs. não ajuste) regulatório sobre as avaliações dos consumidores.

\section{Amostra, design e procedimento}

A amostra foi composta por 149 estudantes de graduação (63,1\% mulheres, idade média de 25,1 anos). O design do estudo 2 foi o mesmo do anterior (dificuldade da tarefa: baixa vs. alta dificuldade) por 2 (foco regulatório: promoção vs. prevenção) por 2 (foco da mensagem: promoção vs. prevenção), between subjects design, tendo sido os respondentes novamente alocados aleatoriamente em uma das oito condições, de forma proporcional.

Para a análise dos resultados, foi utilizado o mesmo procedimento realizado no experimento 1 , com a criação de uma variável de Ajuste vs. Não ajuste, de acordo com as condições de foco regulatório do participante e da mensagem do anúncio.

O experimento 2 seguiu os mesmos procedimentos básicos do experimento 1, com exceção da manipulação do foco regulatório da mensagem do anúncio e da troca da variável dependente por intenção de compra.

Os respondentes, em forma de cover story, foram convidados a participar de três pesquisas não relacionadas. A primeira pesquisa era sobre os Objetivos Acadêmicos e Profissionais; a segunda, sobre a capacidade de resolução de problemas e a terceira, sobre as avaliações de mensagens de anúncios. Todas as pesquisas foram realizadas em sala de aula, com papel e caneta. A duração média de todas as etapas foi de aproximadamente 30 minutos. 
As duas primeiras etapas foram idênticas às realizadas no primeiro estudo. Já para a tarefa de avaliação dos anúncios, foi adaptado o cenário proposto por Cesario, Grant e Higgins (2004), sobre os hábitos alimentares dos estudantes universitários. Uma cover story foi apresentada para explicar que eles iriam avaliar um anúncio para ajudar na elaboração final de uma campanha de incentivo à melhoria de hábitos alimentares para o público alvo.

Aleatoriamente, metade dos participantes recebeu a mensagem com foco em promoção, enfatizando as conquistas e os aspectos positivos de ingerir mais frutas e verduras, enquanto a outra metade recebeu o anúncio na condição de prevenção, que enfatizava a segurança e a vigilância em relação aos hábitos alimentares. Os anúncios são apresentados a seguir:

\section{(Anúncio com foco em Promoção) \\ Consuma mais frutas e verduras e se sinta realizado!}

Uma dieta rica em nutrientes, como os encontrados em frutas e verduras, tem impacto positivo sobre o funcionamento do cérebro, o que resulta em mais energia, bom humor e uma sensação de felicidade e realização. As pessoas que têm uma dieta balanceada, da qual fazem parte frutas e verduras, sentem-se mais confiantes e otimistas. Essas pessoas se mostram mais atraentes, assim como obtêm mais sucesso em seus projetos pessoais e profissionais. O suprimento adequado de nutrientes pela corrente sanguínea também é importante para manter a pele e o cabelo atraentes, além de acelerar o metabolismo, que ajuda a queimar gordura. As vitaminas e os minerais encontrados nas frutas e verduras ajudam a melhorar a concentração e a atenção, o que aumenta as habilidades mentais e a criatividade. Se você tiver uma alimentação saudável diariamente, terá a sensação de bem-estar e se sentirá bem consigo mesmo. (Cesario et al., 2004, p. 391).

\section{(Anúncio com foco em Prevenção)}

\section{Evite consumir frutas e verduras e perca a segurança de ter uma vida saudável!}

As pessoas precisam ter uma dieta rica em nutrientes para ter uma vida saudável. Não ingerir frutas e verduras resulta em falha no suprimento dos nutrientes necessários ao organismo, tornando-o incapaz de produzir substâncias que podem suprir as necessidades de proteção dos fatores negativos que afetam nosso organismo no dia a dia (poluição, estresse, clima, etc.). As vitaminas e minerais encontrados nas frutas e verduras são conhecidos por exercer função de proteção do organismo como um todo. Se você não ingerir frutas e verduras, não ajudará seu sistema imunológico, que não conseguirá mantê-lo saudável e seguro contra doenças. Um sistema imunológico que não é bem nutrido não consegue evitar doenças e impedir a contaminação do organismo por bactérias. Não consumir certos vegetais significa que seu corpo irá perder a oportunidade de obter os nutrientes que são efetivos na proteção do organismo contra câncer e doenças do coração. (Cesario et al., 2004, p. 391).

Depois de ler o anúncio, os respondentes indicavam Qual (seria) sua intenção de comprar mais frutas e verduras após ler a mensagem? $(1=$ nenhuma intenção; 7 = muita intenção). As questões de checagem de manipulação foram as mesmas do experimento 1. Ao todo, cinco respondentes conseguiram adivinhar o propósito da pesquisa associando os estudos (hypothesis guessing check), por isto foram excluídos das análises.

\section{Resultados}

Seguindo os mesmos procedimentos do experimento 1, todos os participantes fizeram de forma adequada a manipulação de foco regulatório de prevenção e promoção conforme a sugestão de Dholakia et al. (2006).

Com relação à manipulação da dificuldade da tarefa, observou-se diferença significativa entre os grupos de acordo com o nível de dificuldade para sua percepção [t $(142)=-11,142, p<0,001$, $\left.\mathrm{M}_{\text {altadificuldade }}=5,44 ; \mathrm{D} \cdot \mathrm{P}=1,51, \mathrm{M}_{\text {baixadificuldade }}=2,63 ; \mathrm{D} \cdot \mathrm{P}=1,52\right]$ e o número de acertos de palavras nos anagramas $\left[\mathrm{t}(142)=13,022, \mathrm{p}<0,001, \mathrm{M}_{\text {baixadificuldade }}=9,21 ; \mathrm{D} \cdot \mathrm{P}=0,99, \mathrm{M}_{\text {altadificuldade }}=4,77 ; \mathrm{D} \cdot \mathrm{P}=2,68\right]$, conforme desejado. 
Não foram encontradas diferenças significativas para envolvimento da tarefa de resolução dos anagramas $\left[\mathrm{t}(142)=0,52, p=0,801, \mathrm{M}_{\text {baixadificuldade }}=5,70 ; \mathrm{D} \cdot \mathrm{P}=1,35, \mathrm{M}_{\text {altadificuldade }}=5,64 ; \mathrm{D} \cdot \mathrm{P}=1,70\right] \mathrm{e}$ avaliação do anúncio [t $(142)=0,619, p=0,537, \mathrm{M}_{\text {baixadificuldade }}=5,01 ; \mathrm{D} \cdot \mathrm{P}=1,57, \mathrm{M}_{\text {altadificuldade }}=4,85$; D.P $=1,58]$ e sua média geral, um pouco maior que no experimento $1(\mathrm{M}=5,30$; $\mathrm{D} . \mathrm{P}=1,28)$, também conforme desejado. Assim, ficou descartada a possível interferência do nível de envolvimento dos participantes com as atividades na interpretação dos resultados.

Para a variável dependente intenção de compra do produto, não foi verificado, na ANOVA, efeito principal do tipo de ajuste $[F(3,140)=5,567 p=0,148]$ ou dificuldade da tarefa $[F(3,140)=0,736, p=$ 0,392]. Entretanto, foi verificada interação significativa entre a dificuldade da tarefa e o tipo de ajuste $\left[F(3,140)=3,866, p=0,051, \eta_{\mathrm{p}}{ }^{2}=0,027\right.$, Figura 2], conforme esperado, portanto, corroborando a hipótese.

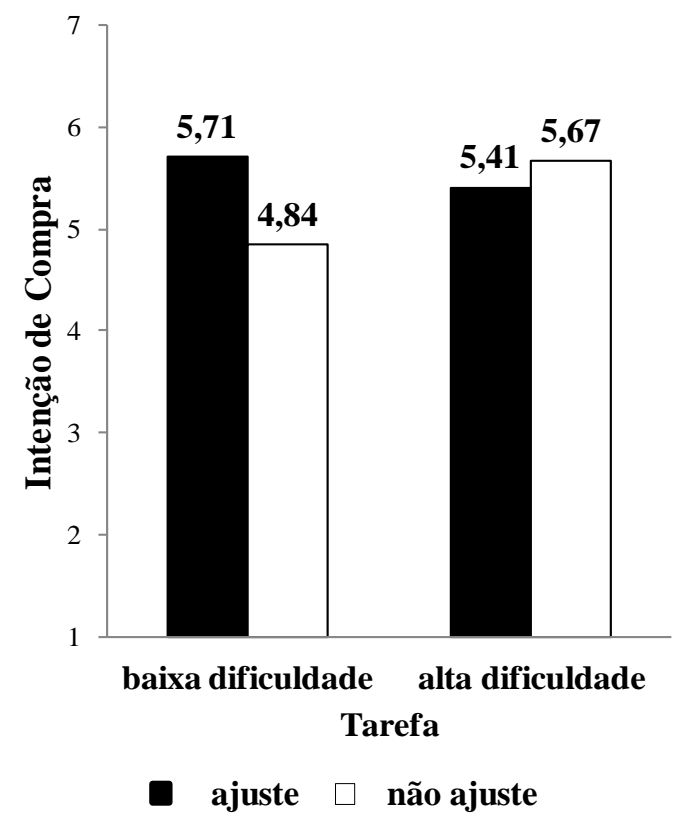

Figura 2. Intenção de Compra em Função da Dificuldade da Tarefa

Comparações post hoc para os grupos de baixa dificuldade apontaram diferença significativa $\left[F(1,69)=5,744, p=0,019, \eta_{\mathrm{p}}^{2}=.078\right]$, com média maior de intenção de compra, para o grupo de ajuste $(\mathrm{M}=5,71$; D.P $=1,62)$, em relação ao de não ajuste $(M=4,84$; D.P $=1,55)$ (lado esquerdo da Figura 2). O mesmo efeito de significância não foi observado dentro do grupo de alta dificuldade $[F(1,73)=$ $0,133, p=.716]$ nas condições de ajuste $(\mathrm{M}=5,41$; $\mathrm{D} . \mathrm{P}=1,70)$ e não ajuste $(\mathrm{M}=5,67$; $\mathrm{D} \cdot \mathrm{P}=1,42)$, ainda que as médias estejam na direção desejada.

Ao avaliar os grupos pelo tipo de ajuste, não foi verificada diferença significativa dentro do grupo de ajuste $[F(1,75)=0,582, p=0,448]$ (barras preenchidas na Figura 2). Porém, dentro do grupo de não ajuste (barras não preenchidas na figura 2), a diferença foi estatisticamente significativa $[F(1,65)=$ $\left.4,340, p=0,041, \eta_{\mathrm{p}}{ }^{2}=0,062\right]$. Os participantes na condição de não ajuste apresentaram maior intenção de compra quando resolveram anagramas considerados mais difíceis $(M=5,67$; D.P $=1,42)$, em relação àqueles que resolveram os anagramas mais fáceis $(\mathrm{M}=4,84$; D.P $=1,55)$. Conforme esperado, houve aumento da intenção de compra na condição de não ajuste quando há alta dificuldade da tarefa.

\section{Discussão}

Os resultados do experimento 2 replicaram de maneira adequada os resultados do experimento 1 . Tanto para a persuasão (experimento 1) quanto para a intenção de compra (experimento 2), o tipo de ajuste teve seu efeito moderado pelo priming de dificuldade da tarefa, com a baixa dificuldade apontando um impacto positivo sobre o ajuste, e a alta dificuldade, um impacto positivo sobre o não ajuste. 
Diversos estudos descreveram o papel do ajuste regulatório em persuasão e situações de compra (Aaker \& Lee, 2001; Avnet et al., 2013; Avnet \& Higgins, 2006; Cesario et al., 2004; Pham \& Avnet, 2009). Porém, as questões pertinentes ao não ajuste regulatório, também em situações de escolhas, ainda carecem de mais aprofundamentos teóricos, e este estudo contribui neste sentido.

Estudos anteriores, como por exemplo os de Harding et al. (2009), verificaram que, em condições de baixo envolvimento, as decisões e avaliações dos consumidores requerem baixo esforço cognitivo. Assim, as pessoas procuram informações e produtos que se ajustem ao seu foco regulatório. Entretanto, quando os indivíduos são motivados a processar informação, o efeito do ajuste é dissipado (J. Wang \& Lee, 2006), sendo uma limitação da condição de ajuste regulatório.

O presente estudo apresentou uma contribuição teórica adicional para explicar em quais condições o ajuste e o não ajuste regulatório influenciam nas escolhas e avaliações subsequentes do consumidor. Dessa forma, este estudo propõe que em determinadas situações, como por exemplo, as de dificuldade de realização de alguma tarefa, os indivíduos são previamente motivados a processar mais informações. Nestes casos, o não ajuste regulatório tem influência mais positiva sobre as avaliações dos consumidores. Por outro lado, em situações de baixa dificuldade da tarefa, nas quais o consumidor não precisa processar muitas informações para tomar uma decisão, o ajuste regulatório é o caminho mais fácil para decidir e, por isso, terá efeito mais positivo sobre as avaliações subsequentes.

O indivíduo com determinado estado de motivação irá captar de maneira mais fácil, nas situações subsequentes, mensagens que estejam de acordo com seu estado de motivação (Aaker \& Lee, 2006; Avnet et al., 2013; Higgins \& Scholer, 2009; Latimer et al., 2007).

Assim, os consumidores mais preocupados em evitar resultados negativos, portanto, em estado de prevenção, serão mais persuadidos por mensagens que passem a ideia de que esse objetivo será atingido. Da mesma forma, aqueles consumidores voltados para alcançar resultados positivos serão mais facilmente influenciados por mensagens congruentes com essa ideia. Porém, isso ocorre somente quando os indivíduos não são estimulados a processar informações. Isso ocorre porque, no dia a dia, as pessoas procuram facilitar suas decisões, conforme mostra o estudo de Bettman, Luce e Payne (1998).

Os resultados deste segundo experimento mostram exatamente este padrão de comportamento. $\mathrm{Na}$ condição de baixa dificuldade da tarefa, a intenção de compra foi bem maior para o grupo de ajuste regulatório $(M=5,71)$ em relação ao de não ajuste $(M=4,84)$.

Os resultados também são consistentes com a proposta dos estudos de Vaughn et al. (2006) e Harding et al. (2009). Estes estudos sugerem que, ao empregarem maior esforço cognitivo, os consumidores ficam mais atentos às informações contrárias ao seu foco motivacional. Neste caso, se estiverem com foco em evitar resultados negativos (prevenção), informações apresentadas com foco em promoção, como por exemplo, Consuma mais frutas e verduras e se sinta realizado, serão mais bem avaliadas. Isso ocorre devido ao estímulo em processar novas informações. No presente estudo, verificou-se que esse padrão de comportamento é verdadeiro. Os consumidores na condição de não ajuste apresentaram maior intenção de compra quando resolveram anagramas considerados mais difíceis $(\mathrm{M}=5,67$; D.P $=1,42)$ em relação àqueles que resolveram os anagramas mais fáceis $(\mathrm{M}=4,84 ; \mathrm{D} . \mathrm{P}=$ $1,55)$.

Pode-se argumentar que a dificuldade da tarefa dos anagramas não foi associada de maneira direta à avaliação das situações de consumo apresentadas nos cenários utilizados. A princípio, essa parece ser uma limitação do estudo, pois se pode pensar em um processo de escolha do produto ou serviço em um cenário de alta dificuldade (comprar um apartamento, por exemplo).

Contudo, o objetivo deste estudo é mostrar o efeito que um priming de dificuldade de avaliação pode ter em avaliações subsequentes de consumo, e isso independe do fato de a dificuldade ser associada diretamente à situação de consumo ou a comportamentos subsequentes. $O$ fato verificado é que $\mathrm{o}$ consumidor, ao ser motivado a processar mais informações, apresentará um comportamento de consumo diferente em termos de ajuste (vs. não ajuste) regulatório. 
Além disso, estudos que procuram aplicar teorias advindas da psicologia social em situações de consumo, normalmente utilizam manipulações de variáveis independentes que, não necessariamente, são apresentadas em uma situação real de consumo. Em especial, o uso de anagramas como tarefa para despertar certos tipos de comportamentos é bastante comum em estudos na área de comportamento do consumidor (ver, por exemplo, C. Wang \& Mukhopadhyay, 2012; Fishbach, Eyal, \& Filkenstein, 2010).

\section{Considerações Finais}

O propósito deste estudo foi melhorar o conhecimento sobre os diferentes mecanismos subjacentes ao efeito do ajuste regulatório sobre a persuasão. $\mathrm{O}$ ajuste regulatório é explicado pelo feeling right (Pham \& Avnet, 2009; Pham et al., 2012). Porém, esse mecanismo é considerado mais abrangente pela literatura, e estudos recentes enfatizam a necessidade de identificar padrões mais exatos por meio dos quais o ajuste regulatório influencia na persuasão (Avnet et al., 2013; Higgins, 2012).

Assim, a contribuição teórica principal deste estudo é identificar um moderador para explicar em quais condições o ajuste e o não ajuste têm maior poder de persuasão sobre os consumidores.

Os resultados dos dois experimentos evidenciam o papel moderador dos diferentes níveis de dificuldade sobre a persuasão e sobre as intenções de compra, tendo como base anúncios de ajuste (vs. não ajuste) regulatório. Em condições de alto envolvimento, os apelos de ajuste regulatório são mais persuasivos quando a dificuldade é baixa e, neste caso, o caminho mais fácil para o consumidor se decidir é pela experiência de feeling right (Pham \& Avnet, 2009). Por outro lado, apelos de não ajuste são mais persuasivos quando os consumidores são motivados a processar mais informações, como por exemplo, em situações de alta dificuldade.

Além disso, a discussão teórica sobre o tipo de ajuste regulatório e os níveis de dificuldade da tarefa e sua posterior constatação empírica podem apresentar contribuições relevantes para a Teoria do Foco Regulatório e principalmente sobre seu papel em situações de consumo.

Sob o ponto de vista prático, em um momento em que a proliferação das informações é instantânea, a capacidade de controle da empresa sobre o comportamento de seu consumidor é reduzida. Assim, a contribuição gerencial deste estudo vai ao encontro das necessidades dos gestores, apontadas pelo Marketing Science Institute (MSI, 2012-2014).

Membros do MSI estão conscientes de que uma única mensagem ou anúncio utilizado para múltiplos estágios, no processo de compra, é ineficiente, pois os consumidores procuram benefícios que estejam em consonância com suas motivações. Assim, faz-se necessário estudar novas estratégias de comunicação para as empresas, na busca por mensagens e anúncios que facilitem o processo de tomada de decisão dos consumidores (MSI, 2012-2014).

Assim, este estudo é relevante na medida em que avança ainda mais para o entendimento do ajuste e do não ajuste regulatório e de sua relação com a dificuldade da tarefa (baixa vs. alta) sobre a avaliação de anúncios de consumo e intenções de compra. Essa compreensão oferece subsídios para que os gestores de marketing desenvolvam estratégias mais efetivas em termos de comunicação com o consumidor, e não sendo reféns dos resultados de ajuste e não ajuste, o gestor pode antecipá-los e induzir também diferentes níveis de dificuldade.

Por exemplo, imagine a compra de um bem relativamente caro, como um carro ou um apartamento, e nesta situação a dificuldade natural de escolher a melhor opção fará com que o consumidor processe o máximo de informações possíveis, uma vez que é uma decisão que influenciará não apenas na sua vida, mas na de outras pessoas, além de ser uma decisão com consequências de longo prazo. 
Em situações como esta, de forma tradicional, o gestor deveria manipular condições de não ajuste entre a orientação regulatória do indivíduo e a orientação das mensagens informativas recebidas (Harding et al., 2009). Ao planejar modos de reduzir o processamento cognitivo (facilitar a tarefa) ou aumentá-lo (dificultar a tarefa), os gerentes podem ter resultados melhores - e de maneira mais fácil.

Os gestores de marketing poderão ainda criar estratégias de anúncios (de carros, de apartamentos, de joias, etc.) voltados para o foco regulatório promocional, e anúncios voltados ao foco regulatório preventivo, com o objetivo de direcionar e segmentar as comunicações, de modo a promover o ajuste ou o não ajuste do comportamento do consumidor, gerando impactos diretos para as compras e recompras ao longo do tempo.

A abordagem contextual que este estudo emprega pode oferecer subsídios para que os gestores de marketing desenvolvam estratégias mais efetivas em termos de comunicação com o consumidor, permitindo que as empresas considerem situações que direcionam as avaliações que os consumidores fazem em contextos de consumo, tais como a atitude do consumidor com relação a um produto, a capacidade de persuasão de uma mensagem, entre outras.

Outro exemplo de estratégia de comunicação é uma das campanhas publicitárias recentes do refrigerante Coca-Cola ${ }^{\circledR}$, que foca nos benefícios e ganhos ao ingerir a bebida. A mensagem é: $\mathbf{1 2 3}$ calorias de energia positiva. Trata-se de uma orientação voltada ao foco regulatório promocional, que poderia, no contexto deste estudo, ser trabalhada dentro dos aspectos da teoria do ajuste (vs. não ajuste) regulatório, deixando a mensagem simples, fácil de ser entendida, melhorando os resultados em ajuste.

Deve ser considerado que, embora a opção metodológica experimental seja a mais adequada para o objetivo deste estudo, suas limitações metodológicas advêm, sobretudo, das características do método selecionado. Nesse sentido, a maior limitação do presente estudo está relacionada à necessidade de controles das variáveis exógenas. O estudo foi realizado em situação de laboratório e contou somente com a participação de universitários.

Contudo, é importante ressaltar que o método experimental busca principalmente a validação interna e, portanto, a generalização para demais contextos de análise não é uma exigência metodológica; no entanto, é sabido que ela mesma poderia trazer maior consistência aos resultados obtidos.

Futuras pesquisas poderiam analisar outras fontes de estímulo ao processamento de informações, como por exemplo, a questão das habilidades e/ou experiência dos sujeitos frente à tarefa realizada, o impacto de tempo de resolução da tarefa, ou mesmo o cansaço (ego-depletion), e como essas relações interferem na avaliação de anúncios de consumo. Ainda, o efeito do desempenho da tarefa não foi considerado sobre as avaliações subsequentes, o que pode ser analisado em estudos futuros.

Apesar de a hipótese ter sido confirmada, ainda há necessidade de confirmar sua consistência em situações de consumo que sejam mais próximas da realidade. Uma possibilidade seria que os respondentes pudessem comprar o produto e efetivamente pagar por ele. Assim, sugere-se um estudo aplicado a um contexto real de consumo, no sentido de verificar a consistência dos resultados obtidos.

\section{Referências}

Aaker, J. L., \& Lee, A. Y. (2001). "I" seek pleasures and "we" avoid pains: the role of self-regulatory goals in information processing and persuasion. Journal of Consumer Research, 28(6), 33-49. doi: $10.1086 / 321946$

Aaker, J. L., \& Lee, A. Y. (2006). Understanding regulatory fit. Journal of Marketing Research, 43(1), 15-19. doi: 10.1509/jmkr.43.1.15 
Appelt, K. C., Zou, X., \& Higgins, E. T. (2010). Feeling right or being right: when strong assessment yields strong correction. Motivational Emotional, 34(3), 316-324. doi: 10.1007/s11031-0109171-z

Avnet, T., \& Higgins, E. T. (2006). How regulatory fit affects value in consumer choices and opinions. Journal of Marketing Research, 43(1), 1-10. doi: 10.1509/jmkr.43.1.1

Avnet, T., Laufer, D., \& Higgins, E. T. (2013). Are all experiences of fit created equal? Two paths to persuasion. Journal of Consumer Psychology, 23(3), 301-316. doi: 10.1016/j.jcps.2012.10.011

Avnet, T., Pham, M. T., \& Stephen, A. T. (2012). Consumers' trust in feeling as information. Journal of Consumer Research, 39(4), 720-735. doi: 10.1086/664978

Bettman, J. R., Luce, M. F., \& Payne, J. W. (1998). Constructive consumer choice processes. Journal of Consumer Research, 25(3), 187-217. doi: 10.1086/209535

Cesario, J., Grant, H., \& Higgins, E. T. (2004). Regulatory fit and persuasion? Transfer from "feeling right". Journal of Personality and Social Psychology, 86(3), 388-404. doi: 10.1037/00223514.86.3.388

Cesario, J., \& Higgins, E. T. (2008). Making messages recipients "feel right" - how nonverbal cues can increase persuasion. Psychological Science, 19(5), 415-420. doi: 10.1111/j.14679280.2008.02102.x

Chernev, A. (2004). Goal-attribute compatibility in consumer choice. Journal of Consumer Psychology, 14(1/2), 141-150. doi: 10.1207/s15327663jcp1401\&2_16

Dholakia, U. M., Gopinath, M., Bagozzi, R. P., \& Nataraajan, R. (2006). The role of regulatory focus in the experience and self-control of desire for temptations. Journal of Consumer Psychology, 16(2), 163-175. doi: $10.1207 / \mathrm{s} 15327663 j \mathrm{jcp} 1602 \_7$

Faro, D., Heller, M., \& Irmak, C. (2011, November). Merely available: products may be effective without actual consumption. Proceedings of the Society for Judgment and Decision Making Conference, Seattle, WA, USA, 32.

Fishbach, A., \& Dhar, R. (2005). Goals as excuses or guides: the liberating effect of perceived goal progress on choice. Journal of Consumer Research, 32(3), 370-377. doi: doi.org/10.1086/497548

Fishbach, A., Eyal, T., \& Finkelstein, S. R. (2010). How positive and negative feedback motivate goal pursuit. Social and Personality Psychology Compass, 4(8), 517-530. doi: 10.1111/j.17519004.2010.00285.x

Florack, A., Ineichen, S., \& Bieri, R. (2009). The impact of regulatory focus on the effects of two-sided advertising. Social Cognition, 27(1), 37-56. doi: 10.1521/soco.2009.27.1.37

Forster, J., Higgins, E. T., \& Werth, L. (2004). How threat from stereotype disconfirmation triggers selfdefense. Social Cognition, 22(1), 54-74. doi: 10.1521/soco.22.1.54.30982

Harding, L. M., Lisjak, M., \& Lee, A. Y. (2009, October). Persuasive power of regulatory nonfit. Proceedings of the Association for Consumer Research Conference, Pittsburgh, PA, USA, 36.

Haws, K. L., Dholakia, U. M., \& Bearden, W. O. (2010). An assessment of chronic regulatory focus measures. Journal of Marketing Research, 47(5), 967-982. doi: 10.1509/jmkr.47.5.967

Higgins, E. T. (1997). Beyond pleasure and pain. American Psychologist, 52(12), 1280-1300. doi: 0003066X.52.12.1280 
Higgins, E. T. (2002). How self-regulation creates distinct values: the case of promotion and prevention decision making. Journal of Consumer Psychology, 12(3), 177-191. doi: 10.1207/S15327663JCP1203_01

Higgins, E. T. (2004). Regulatory fit: an experience that creates value. Journal of Cultural and Evolutionary Psychology, 2(1/2), 9-22. doi: 10.1556/JCEP.2.2004.1-2.1

Higgins, E. T. (2012). Beyond pleasure and pain: how motivation works. New York, NY: Oxford University Press.

Higgins, E. T., Friedman, R. S., Harlow, R. E., Idson, L. C., Ayduk, O. N., \& Taylor, A. (2001). Achievement orientation from subjective histories of success: promotion pride versus prevention pride. European Journal of Social Psychology, 31(1), 3-23. doi: 10.1002/ejsp.27

Higgins, E. T., \& Scholer, A. A. (2009). Engaging the consumer: the science and art of the value creation process. Journal of Consumer Psychology, 19(2), 100-114. doi: 10.1016/j.jcps.2009.02.002

Laran, J., Janiszewski, C., \& Cunha, M., Jr. (2008). Context-dependent effects of goal primes. Journal of Consumer Research, 35(4), 653-667. doi: 10.1086/592127

Latimer, A. E., Rivers, S. E., Rench, T. A., Katulak, N. A., Hicks, A., Hodorowski, J. K., Higgins, E. T., \& Solovey, P. (2008). A field experiment testing the utility of regulatory fit messages for promoting physical activity. Journal of Experimental Social Psychology, 44(3), 826-832. doi: 10.1016/j.jesp.2007.07.013

Lee, A. Y. (2009). Engaging the consumer: the opposing forces of regulatory nonfit versus fit. Journal of Consumer Psychology, 19(2), 134-136. doi: 10.1016/j.jcps.2009.02.006

Lee, A. Y., \& Aaker, J. L. (2004). Bringing the frame into focus: the influence of regulatory fit on processing fluency and persuasion. Journal of Personality and Social Psychology, 86(2), 205218. doi: 10.1037/0022-3514.86.2.205

Lee, A. Y., Keller, P. A., \& Sternthal, B. (2010). Value from regulatory construal fit: the persuasive impact of fit between consumer goals and message concreteness. Journal of Consumer Research, 36(5), 735-747. doi: 10.1086/605591

Mogilner, C., Aaker, J. L., \& Pennington, G. L. (2008). Time will tell: the distant appeal of promotion and imminent appeal of prevention. Journal of Consumer Research, 34(5), 670-681. doi: $10.1086 / 521901$

Nordgren, L. F., \& Dijksterhuis, A. (2009). The devil is in the deliberation: thinking too much reduces preference consistency. Journal of Consumer Research, 36(1), 39-46. doi: 10.1086/596306

Norris, M. E. (2007). The impact of information processing goals and capacity restrictions (Doctoral dissertation). Queen's University, Kingston, Ontario, Canada.

Pham, M. T., \& Avnet, T. (2009). Rethinking regulatory engagement theory. Journal of Consumer Psychology, 19(2), 115-123. doi: 10.1016/j.jcps.2009.02.003

Pham, M. T., \& Chang, H. H. (2010). Regulatory focus, regulatory fit, and the search and consideration of choice alternatives. Journal of Consumer Research, 37(4), 626-640. doi: 10.1086/655668

Pham, M. T., Lee, L., \& Stephen, A. T. (2012). Feeling the future: the emotional oracle effect. Journal of Consumer Research, 39(3), 461- 477. doi: 10.1086/663823

Reinhard, M. A., \& Dickhauser, O. (2011). How affective states, task difficulty, and self-concepts influence the formation and consequences of performance expectancies. Cognition and Emotion, 25(2), 220-228. doi: 10.1080/02699931003802640. 
Rice, S., Geels, K., Hackett, H. R., Trafimow, D., McCarley, J., Schwark, J., \& Hunt, G. (2012). The harder the task, the more inconsistent the performance: a PPT analysis on task difficulty. The Journal of General Psychology, 139(1), 1-18. doi: 10.1080/00221309.2011.619223.

Shadish, W. R., Cook, T. D., \& Campbell, D. T. (2002). Experimental and quasi-experimental designs for generalized causal inference. New York: Houghton Mifflin Company.

Tam, L., \& Spanjol, J. (2012). When impediments make you jump rather than stumble: regulatory nonfit, implementation intentions and goal attainment. Marketing Letters, 23(1), 93-107. doi: 10.1007/s11002-011-9138-1

Vaughn, L. A., O’Rourke, T., Schwartz, S., Malik, J., Petkova, Z., \& Trudeau, L. (2006). When two wrongs can make a right: regulatory nonfit, bias, and correction of judgments. Journal of Experimental Social Psychology, 42(5), 654-661. doi: 10.1016/j.jesp.2005.09.004

Wang, C., \& Mukhopadhyay, A. (2012). The dynamics of goal revision: a cybernetic multiperiod testoperate-test-adjust-loop (total) model of self-regulation. Journal of Consumer Research, 38(5), 815-832. doi: 10.1086/660853

Wang, J., \& Lee, A. Y. (2006). The role of regulatory focus in preference construction. Journal of Marketing Research, 43(1), 23-38. doi: 10.1509/jmkr.43.1.28

Werth, L., \& Foerster, J. (2007). How regulatory focus influence consumer behavior. European Journal of Social Psychology, 37(1), 33-51. doi: 10.1002/ejsp.343

\title{
Dados dos Autores
}

\author{
Danielle Mantovani \\ Av. Prof. Lothario Meissner, 632, Jardim Botânico, 80210-170, Curitiba, PR, Brasil. E-mail: dm_lucena@yahoo.com.br \\ Mariana Monfort Barboza \\ Av. Prof. Lothario Meissner, 632, Jardim Botânico, 80210-170, Curitiba, PR, Brasil. E-mail: marianamonfort@ gmail.com \\ Juan José Camou Viacava \\ Av. Prof. Lothario Meissner, 632, Jardim Botânico, 80210-170, Curitiba, PR, Brasil. E-mail: juanjcviacava@ gmail.com \\ Paulo Henrique Muller Prado \\ Av. Prof. Lothario Meissner, 632, Jardim Botânico, 80210-170, Curitiba, PR, Brasil. E-mail: pprado@ufpr.br
}

